\title{
Internal carotid artery injury in the endoscopic transsphenoidal surgery for pituitary adenoma: an uncommon case and literature review
}

\author{
Xiaohai Liu ${ }^{1}$, Ming Feng ${ }^{2}$, Congxin $\mathrm{Dai}^{2}$, Xinjie $\mathrm{Bao}^{2}$, Kan Deng ${ }^{2}$, Yong $\mathrm{Yao}^{2}$, Renzhi Wang ${ }^{2}$ \\ ${ }^{1}$ Department of Neurosurgery, Xuanwu Hospital Capital Medical University, Beijing, China; ${ }^{2}$ Department of Neurosurgery, Peking Union Medical \\ College Hospital, Chinese Academy of Medical Sciences and Peking Union Medical College, Beijing, China \\ Correspondence to: Renzhi Wang. Department of Neurosurgery, Peking Union Medical College Hospital, Chinese Academy of Medical Sciences and \\ Peking Union Medical College, Beijing, China. Email: wangrz@126.com.
}

\begin{abstract}
Complications of the transsphenoidal surgery (TSS) for pituitary adenoma (PA) include vascular injury, nerve injury and nervous system infection. Among these, the consequences of internal carotid artery (ICA) injury are the most serious, and its mortality and disability rates are very high. The TSS was used in 889 patients with PA, among which there was 1 case of ICA injury. In this uncommon case of bromocirptineresistant macro-prolatinoma, the patient had received an ultrahigh dose of oral bromocriptine for almost three years and was intraoperatively found to have a tenacious tumor texture and close adhesion of the tumor capsule to the ICA. Resection of the tumor capsule required excessive force, resulting in the rupture at the A1 origin of the left ICA. Endovascular balloon occlusion was used after urgent angiography. The patient died in two weeks even with intensive treatment. Although the underlying mechanism is still obscure, bromocriptine-inducing tumor fibrosis in PA is relatively common. For these patients with excessive dose of bromocriptine, tumor fibrosis and ICA injury should be seriously considered and performing a complete preoperative imaging evaluation to achieve accurate intraoperative positioning and carefully performing the operation are needed to preventing ICA injury. In some cases, resection of the tumor pseudocapsule should not be forced to avoid ICA injury.
\end{abstract}

Keywords: Pituitary adenoma; endoscopic transsphenoidal approach; internal carotid artery injury; bromocriptine; case report

Submitted Mar 15, 2020. Accepted for publication Jun 17, 2020.

doi: 10.21037 /gs-20-354

View this article at: http://dx.doi.org/10.21037/gs-20-354

\section{Introduction}

The sellar area is a common location for nervous system tumors, with pituitary adenoma (PA) being the most common tumor in this region. With the exception of prolactinomas, which can be cured or controlled by dopamine agonists, the standard treatment for PAs is transsphenoidal surgery (TSS), which was established in the early 20th century (1). As endoscopic techniques have rapidly improved, endoscopy-assisted TSS can achieve maximum exposure of the saddle area and the area surrounding the structures at the skull base and is currently used to treat various conditions, including invasive PAs, craniopharyngiomas, and Rathke's cysts, with good therapeutic efficacy (2). However, as the anatomical structures in the sellar region are complex and prone to variation, collateral damage to important surrounding structures, such as vascular injury and nerve damage, may occur when using the extended TSS for invasive tumors. Among these complications, internal carotid artery (ICA) injury, with an incidence ranging from $0 \%$ to $0.1 \%$, has the most serious consequences, with very high mortality and disability rates, leading to a catastrophic prognosis in PA patients undergoing treatment with the TSS (3). However, there is no concise standard guidelines on how to manage 

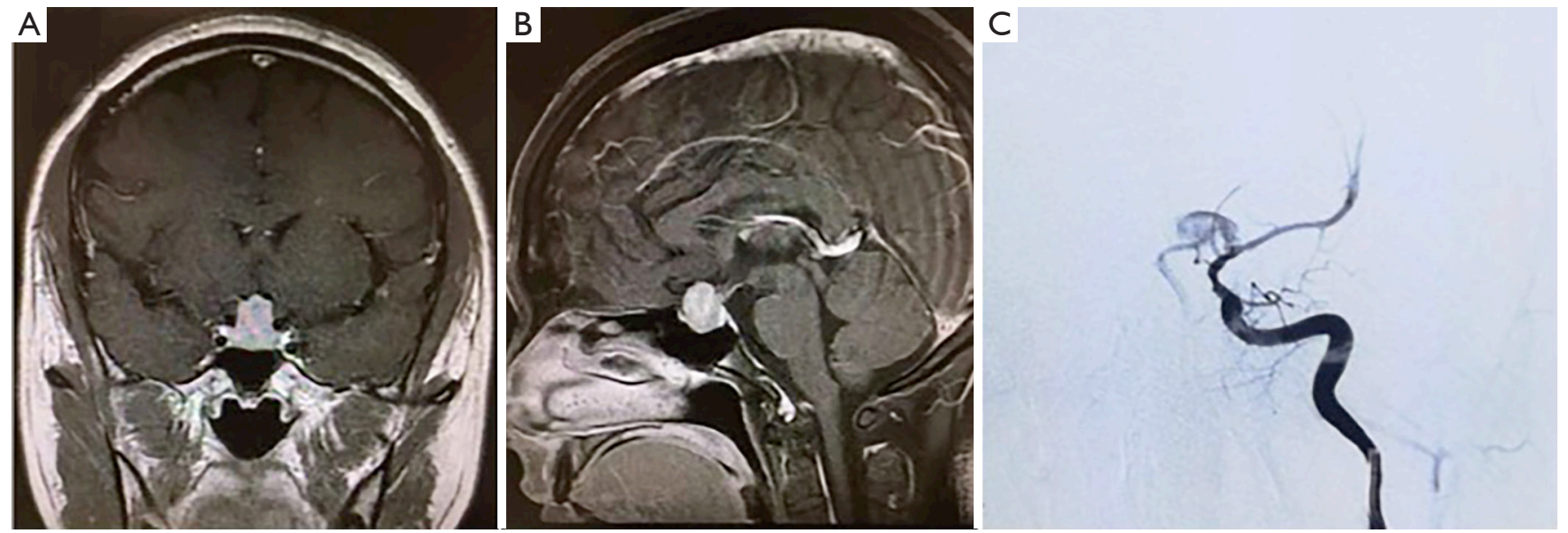

Figure 1 The tumor in MRI and the rupture in DSA. (A and B) Enhanced magnetic resonance imaging revealed a pituitary macroadenoma. (C) Cerebral angiography showed a rupture at the A1 origin of the left ICA. ICA, internal carotid artery.

the ICA injury during TSS.

In this study, we reported an uncommon case of ICA injury during TSS in a prolactinoma patient with ultrahigh dose of long-term oral bromocriptine-induced tumor fibrosis and a fatal outcome. Although the underlying mechanism is still obscure, bromocriptine-inducing tumor fibrosis in PA is relatively common. For these patients with excessive dose of bromocriptine, tumor fibrosis and ICA injury should be seriously considered. Performing a complete preoperative imaging evaluation to achieve accurate intraoperative positioning and carefully performing the operation are needed to preventing ICA injury. We present the following case in accordance with the CARE reporting checklist.

\section{Case presentation}

From January 2016 to December 2018, a total of 889 patients underwent endoscopic TSS in the Department of Neurosurgery, Peking Union Medical College Hospital. During this period, a total of 1 case of intraoperative ICA injury occurred. The patient was a 31-year-old male, presented with decreased libido, erectile dysfunction and headache that occurred approximately 1-2 times per day and was not relieved by rest. His visual fields were relatively normal. He was diagnosed with hyperprolactinemia and macro-prolactinoma based on magnetic resonance imaging and an increased prolactin level of approximately $310 \mathrm{ng} / \mathrm{mL}$ in the local hospital. Then the patient was treated with bromocriptine at $30 \mathrm{mg}$ per day for almost 3 years. Routine follow-up showed that the tumor was still enlarged and that the prolactin level had progressively increased to $398 \mathrm{ng} / \mathrm{mL}$. The symptoms such as decreased libido, erectile dysfunction and headache was not improve but aggravated. Moreover, side effects from excessive bromocriptine, such as dizziness, nausea and vomiting, and weakness seriously affected his work efficiency and quality of life. In May 2018, he was admitted to the outpatient department of our hospital, where his prolactin level was found to be $772 \mathrm{ng} / \mathrm{mL}$, and enhanced magnetic resonance imaging revealed a pituitary macroadenoma (Figure 1A,B). After a multidisciplinary team discussion, a TSS was recommended to remove the PA. Intraoperatively, the tumor was found to be very tough and firm, exhibiting fibrous scarring that blocked resection of the tumor. When we tried to separate the pseudocapsule of the tumor from the diaphragma sellae, sudden bleeding occurred. The bleeding was too heavy to continue the resection, and we used iodoform gauze to achieve hemostasis. Then the cerebral angiography showed a rupture at the A1 origin of the left ICA (Figure 1C). An endovascular balloon was applied to occlude the left ICA as there was no stent available. Then, bilateral lateral ventricle drainage was performed immediately. Postoperatively, the patient was returned to the intensive care unit for treatment. Two weeks after surgery, the patient died because of intracranial ischemic complications. A timeline covering what's done and what's found with an accurate time of each stage was shown in Figure 2.

To investigate the fibrosis of the tumor, $\mathrm{H} \& \mathrm{E}$ staining was used. The tumor sample from another patient with 


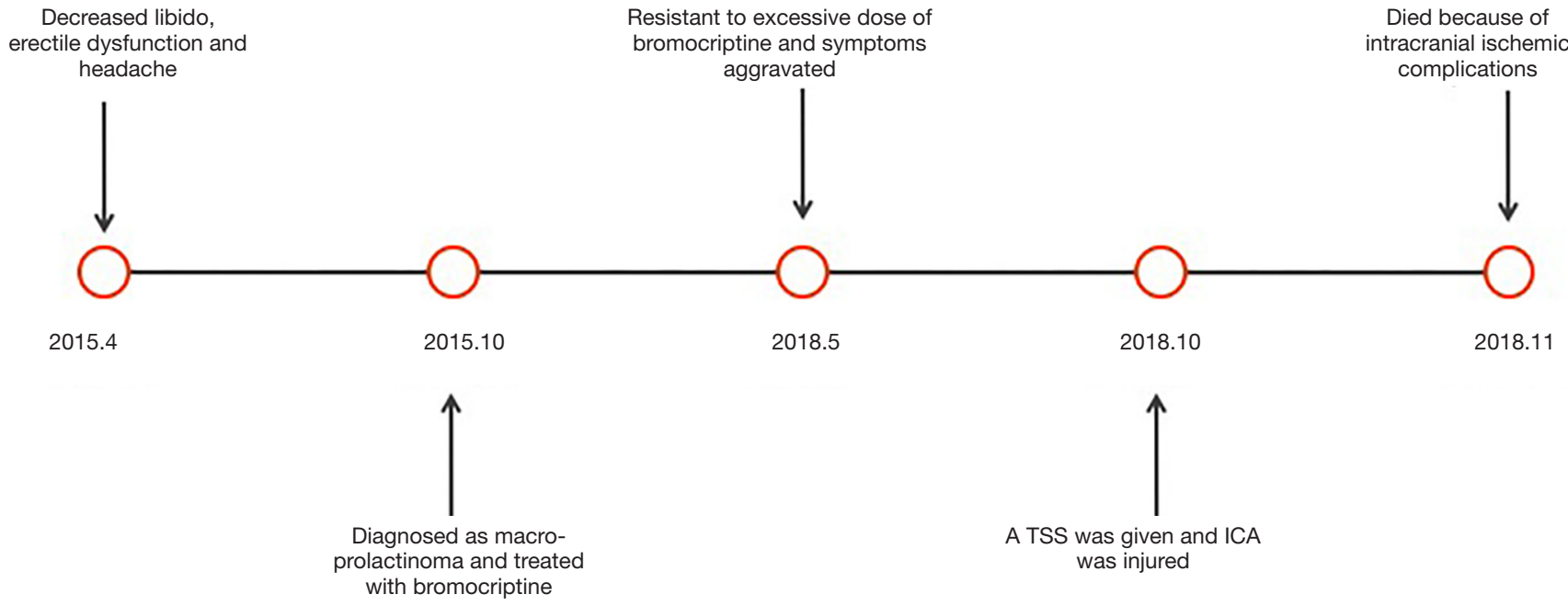

Figure 2 A timeline covering what's done and what's found with an accurate time of each stage was shown.
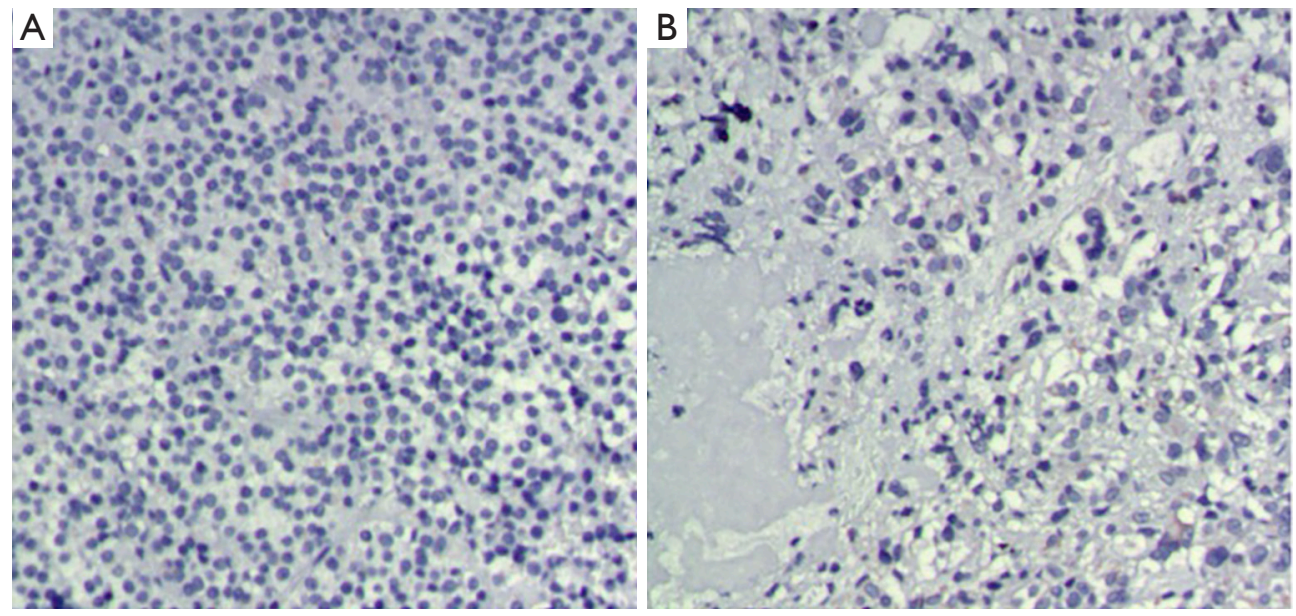

Figure $3 \mathrm{H} \& \mathrm{E}$ staining of the tumor. (A) Nests of prolactinoma (no bromocriptine administration) cells with no or little fibrosis (H\&E, original magnification $\times 200$ ). (B) Dense tumorous fibrosis with diminution of prolactinoma cell $(\mathrm{H} \& \mathrm{E}$, original magnification $\times 200)$.

macro-prolactinoma who refused to take bromocriptine for severe side effects was used as control. The staining showed dense tumorous fibrosis in our patient while no or little fibrosis in the control (Figure 3). Written informed consent was obtained from the patient's wife according to the institutional guidelines.

\section{Discussion}

Although most PAs show benign tumor characteristics, approximately $40 \%$ of these tumors invade the cavernous sinus, even enveloping the ICA and other important structures (4). After years of development, the TSS has replaced craniotomy and become the standard surgical method for PA resection. Since the beginning of the $21 \mathrm{st}$ century, Kassam, Castelnuovo, and Cappabianca have gradually introduced endoscopic extended TSS to remove invasive PA, craniopharyngiomas, meningiomas, and chordomas $(5,6)$. However, due to the complex anatomy of the saddle region, the extended TSS also has a higher risk of vascular and nerve damage. Among these complications, the most serious is injury of the ICA and its important branches, which leads to disastrous consequences if not handled properly (7). 
In this retrospective study, the incidence of ICA injury in TSS for PA was $0.11 \%$, which is consistent with the incidence reported in the literature. May et al. reviewed a total of 4,691 patients and found one case of intraoperative ICA injury (8). Weidenbecher et al. experienced four cases of ICA injury following sphenoidectomy among 16,000 endoscopic sinus procedures performed over a 30 -year period (9). However, the reported mortality rate of this complication is approximately $14 \%$, and its disability rate is approximately $24 \%$ (10). Hence, although its incidence is very low, its mortality and disability rates are very high, and this complication therefore merits more attention.

The possible risk factors related to ICA injury include the location and nature of the lesion, anatomical variation in the saddle region, and the operator's surgical experience. Large PAs often invade the surrounding cavernous sinus and suprasellar structures or erode the skull base, and an extended approach is often adopted. Tumors located in the cavernous sinus, clival regions and suprasellar cisterna carry an increased possibility of damage to the ICA and its branches during resection. The risk of ICA injury is also different for different types of PA. For example, patients with Cushing's disease caused by adrenocorticotropic hormone-secreting PAs often show associated hypertension and atherosclerosis, which increase the likelihood of the ICA being damaged when scratched or pulled during surgery (11). Acromegaly can also cause anatomical changes in the nasal cavity and sphenoid sinus that shorten the ICA and thereby increase the risk of ICA injury (12). Surgery can cause an anatomical disorder in the sellar region or along the original surgical path, resulting in scar formation in the surgical area or the adhesion of important vessels to the tumor; therefore, it is sometimes difficult to distinguish the interface between the tumor tissue and the pituitary gland, and the risk of ICA injury caused by reoperation is also significantly increased in these cases (13). Moreover, intraoperative experience has been significantly correlated with the occurrence of ICA injury. The rate of ICA injury was inversely proportional to the experience of the surgeon for surgeries performed in the sphenoid sinus: the ICA injury rate of the least experienced surgeons (200 cases of TSS) was $1.4 \%$, while that of surgeons with intermediate experience (200-500 cases) was $0.6 \%$, and that of the most experienced surgeons ( $>500$ cases) was even lower, at $0.4 \%$ (14). According to Charalampaki, approximately 100 to 150 cases of TSS are required to accumulate enough experience to produce significant improvement in surgical techniques (15).

Interestingly in this case, the patient with prolactinoma was treated with bromocriptine at $30 \mathrm{mg}$ per day for almost 3 years before the operation. Although the tumor did not invade the ICA or other important branches, long-term oral treatment with an excessive dose of bromocriptine resulted in fibrosis of the PA. The tumor was intraoperatively found to be very tough and firm and showed obvious fibrotic scarring. After resecting most of the tumor in blocks, the attempt to remove the tumor pseudocapsule led to excessive pulling, resulting in tearing and bleeding of the artery. Intraoperative endoscopy failed to allow observation of the rupture; cerebral angiography was promptly applied after oppression hemostasis, showing a rupture at the A1 origin of the left ICA. Even with intensive treatment for two weeks, the patient died of intracranial ischemic complications.

This case is an interesting addition to the risk factors for ICA rupture during TSS by emphasizing bromocriptineinduced fibrosis of prolactinomas. Although dopaminergic agents such as bromocriptine and cabergoline have become the first line treatment for prolactinoma, more and more evidences showed prolactinomas treated with bromocriptine developed tumor fibrosis, which has generated a renewed interest in surgical treatment of prolactinomas, and has promoted some controversy regarding first line therapy (16). Although Cabergoline is currently the preferred first-line treatment option for prolactinomas, it is not available in China while bromocriptine is the only choice. In our case, the patients took excessive dose of bromocriptine, resulting in fibrosis of the tumor, which might be one of the predispositions for ICA rupture. Therefore, in cases with severe tumor fibrosis, which is often seen in patients with preoperative drug therapy and those undergoing reoperation, more attention should be paid when separating the tumor pseudocapsule. Sharp separation is recommended, and excessive pulling should be avoided. The pseudocapsule could be retained when the tumor strongly adheres to the ICA and its branches.

During TSS, the occurrence of ICA injury should be identified immediately, and an appropriate plan for dealing with this potential complication should be devised before the operation (17). If a large amount of intraoperative bleeding occurs, it should be determined as soon as possible whether the source of the bleeding is the ICA or the cavernous sinus, an intercavernous sinus or another venous system. If the color of the blood is deep red, similar to the 
color of venous blood, and the rate of bleeding is much lower than that observed for arterial bleeding, it is bleeding from the cavernous sinus or an intercavernous sinus. In this case, hemostatic material should be used to compress and stop the bleeding first, and tumor resection could be performed without stopping the operation. However, if the blood is bright red and the rate of bleeding is extremely fast, ICA bleeding should be strongly suspected, and the operation should be stopped immediately. While pressing the common carotid artery of the patient, rapid fluid rehydration should be performed, along with nasal filling and compression hemostasis with gelfoam and strips. Direct suturing to stop the bleeding is impossible by endoscopic surgery in this case. By contrast, direct repair using either direct suturing or bipolar coagulation may cause excessive blood loss and unnecessary delays in controlling the bleeding. At the same time, cerebral arteriography should be immediately performed to determine the location, size and relationship between the ICA injury and adjacent structures.

ICA injuries can be divided into three types: ICA rupture and hemorrhage, pseudoaneurysm and internal carotid cavernous sinus fistula (17). Once angiography has identified intraoperative ICA rupture and hemorrhage, it should be treated according to the type of injury. Usually, endovascular stent implantation or balloon embolization is selected. In this case, after balloon embolization, due to the low compensation of the blood supply from the contralateral side, the ipsilateral blood supply was interrupted, and a large-scale cerebral infarction occurred, leading to the patient's death.

According to the different locations in which it may occur, an ICA injury will generally be divided into four modes: ICA injury, anterior cerebral artery and anterior traffic complex injury, ocular artery injury, and posterior cerebral artery injury. Among these, ICA injuries are the most common. In terms of remedial measures, direct endoscopic suturing is very difficult to achieve in cases with a large amount of bleeding, which blurs the surgical field, and attempts at direct repair can lead to excessive blood loss. Therefore, direct suturing of the rupture is not recommended. Bipolar electrocoagulation, similar to direct suturing, is also difficult to perform in cases with massive bleeding, and the risk of delayed pseudoaneurysm after direct cauterization is extremely high. Of the 29 such cases reported in the literature, only 2 were successfully treated with this technique (10). Therefore, at present, the most effective method consists of compression with surgical hemostatic material encapsulation, angiography, and concurrent interventional therapy. Materials such as hemostatic sponges, muscle or fat can be used. Angiography can be used to identify the location of the bleeding and the degree of stenosis after vascular compression and reveal any pseudoaneurysm formation or thrombosis. Possible endovascular treatments include coil embolization, stentassisted coiling, and stent implantation. Primary ICA occlusion is tolerated in most patients (80-87\%). A balloon occlusion test should be considered if the risk of injury is considered to be high preoperatively. For example, Gardner et al. performed balloon occlusion in a tumor surrounding the ICA (18). If the test occlusion fails in the patient, care should be taken not to injure the ICA during surgery (19). The limitation of the case is no stent available at that time where an endovascular balloon was applied to occlude the left ICA. The prognosis of the patient would be much better if a stent was used in this case.

For these patients with excessive dose of bromocriptine, tumor fibrosis and ICA injury should be seriously considered and performing a complete preoperative imaging evaluation to achieve accurate intraoperative positioning and carefully performing the operation are needed to preventing ICA injury. In some cases, resection of the tumor pseudocapsule should not be forced to avoid ICA injury.

\section{Acknowledgments}

Funding: The financial support for this study was provided by the Scientific Research Project of Capital Health Development in 2018 (grant number: 2018-4-4018), CAMS Innovation fund for Medical Science (grant number: CIFMS, 2017-12M-2-005). The funding institutions played no role in the design of the study, data collection or analysis, decision to publish, or preparation of the manuscript.

\section{Footnote}

Conflicts of Interest: All authors have completed the ICMJE uniform disclosure form (available at http://dx.doi. org/10.21037/gs-20-354). The authors have no conflicts of interest to declare.

Ethical Statement: The authors are accountable for all aspects of the work in ensuring that questions related to the accuracy or integrity of any part of the work are appropriately investigated and resolved. All procedures performed in studies involving human participants were 
in accordance with the Declaration of Helsinki (as revised in 2013). Written informed consent was obtained from the patient's wife according to institutional guidelines for publication of this case report and any accompanying images.

Open Access Statement: This is an Open Access article distributed in accordance with the Creative Commons Attribution-NonCommercial-NoDerivs 4.0 International License (CC BY-NC-ND 4.0), which permits the noncommercial replication and distribution of the article with the strict proviso that no changes or edits are made and the original work is properly cited (including links to both the formal publication through the relevant DOI and the license). See: https://creativecommons.org/licenses/by-nc-nd/4.0/.

\section{References}

1. Theodros D, Patel M, Ruzevick J, et al. Pituitary adenomas: historical perspective, surgical management and future directions. CNS Oncol 2015;4:411-29.

2. Wang AJ, Zaidi HA, Laws ED Jr. History of endonasal skull base surgery. J Neurosurg Sci 2016;60:441-53.

3. Berker M, Hazer DB, Yücel T, et al. Complications of endoscopic surgery of the pituitary adenomas: analysis of 570 patients and review of the literature. Pituitary 2012;15:288-300.

4. Li-Ng M, Sharma M. Invasive pituitary adenoma. J Clin Endocrinol Metab. 2008,93:3284-5.

5. Kassam A, Snyderman CH, Mintz A, et al. Expanded endonasal approach: the rostrocaudal axis. Part I. Crista galli to the sella turcica. Neurosurg Focus 2005;19:E3.

6. Kassam A, Snyderman CH, Mintz A, et al. Expanded endonasal approach: the rostrocaudal axis. Part II. Posterior clinoids to the foramen magnum. Neurosurg Focus 2005;19:E4.

7. Valentine R, Wormald PJ. Carotid artery injury after endonasal surgery. Otolaryngol Clin North Am 2011;44:1059-79.

8. May M, Levine HL, Mester SJ, et al. Complications of

Cite this article as: Liu X, Feng M, Dai C, Bao X, Deng K, Yao Y, Wang R. Internal carotid artery injury in the endoscopic transsphenoidal surgery for pituitary adenoma: an uncommon case and literature review. Gland Surg 2020;9(4):1036-1041. doi: $10.21037 / g s-20-354$ endoscopic sinus surgery: Analysis of 2108 patients incidence and prevention. Laryngoscope 1994;104:1080-3.

9. Weidenbecher $M$, Huk WJ, Iro H. Internal carotid artery injury during functional endoscopic sinus surgery and its management. Eur Arch Otorhinolaryngol 2005;262:640-5.

10. Romero ADCB, Lal Gangadharan J, Bander ED, et al. Managing Arterial Injury in Endoscopic Skull Base Surgery: Case Series and Reviewof the Literature. Oper Neurosurg (Hagerstown) 2017;13:138-49.

11. Oskouian RJ, Kelly DF, Laws ERJ Jr. Vascular injury and transsphenoidal surgery. Front Horm Res 2006;34:256-78.

12. Ebner FH, Kuerschner V, Dietz K, et al. Reduced intercarotid artery distance in acromegaly: pathophysiologic considerations and implications for transsphenoidal surgery. Surg Neurol 2009;72:456-60.

13. Saeger W, Muller M, Buslei R, et al. Recurrences of Pituitary Adenomas or Second De Novo Tumors: Comparisons with First Tumors. World Neurosurg 2018;119:e118-24.

14. Ciric I, Ragin A, Baumgartner C, et al. Complications of transsphenoidal surgery: results of a national survey, review of the literature, and personal experience. Neurosurgery 1997;40:225-36.

15. Charalampaki P, Ayyad A, Kockro RA, et al. Surgical complications after endoscopic transsphenoidal pituitary surgery. J Clin Neurosci 2009;16:786-9.

16. Menucci M, Quiñones-Hinojosa A, Burger P, et al. Effect of dopaminergic drug treatment on surgical findings in prolactinomas. Pituitary 2011;14:68-74.

17. Dusick JR, Esposito F, Malkasian D, et al. Avoidance of carotid artery injuries in transsphenoidal surgery with the Doppler probe and micro-hook blades. Neurosurgery 2007;60:322-8.

18. Gardner PA, Tormenti MJ, Pant H, et al. Carotid artery injury during endoscopic endonasal skull base surgery: incidence and outcomes. Neurosurgery 2013;73:ons261-9.

19. Regmi D, Thapa A, Kc B, et al. Endoscopic Endonasal Transsphenoidal Approach to Pituitary Adenoma: A Multi-disciplinary Approach. J Nepal Health Res Counc 2017;15:174-7. 